\title{
Analyzing the Influence of Landscape Aggregation on Disease Spread to Improve Management Strategies
}

\author{
Coralie Picard,1 Samuel Soubeyrand,2 Emmanuel Jacquot, ${ }^{1}$ and Gaël Thébaudd, ${ }^{1,}$ \\ ${ }^{1}$ BGPI, INRA, Montpellier SupAgro, Univ Montpellier, Cirad, TA A-54/K, 34398, Montpellier Cedex 5, France \\ ${ }^{2}$ BioSP, INRA, Avignon, France \\ Accepted for publication 1 February 2019.
}

\begin{abstract}
Epidemiological models are increasingly used to predict epidemics and improve management strategies. However, they rarely consider landscape characteristics although such characteristics can influence the epidemic dynamics and, thus, the effectiveness of disease management strategies. Here, we present a generic in silico approach which assesses the influence of landscape aggregation on the costs associated with an epidemic and on improved management strategies. We apply this approach to sharka, one of the most damaging diseases of Prunus trees, for which a management strategy is already applied in France. Epidemic simulations were carried out with a spatiotemporal stochastic model under various management strategies in landscapes differing in patch aggregation. Using sensitivity analyses, we highlight the impact of management parameters on the economic output of the model. We also show that the sensitivity analysis

can be exploited to identify several strategies that are, according to the model, more profitable than the current French strategy. Some of these strategies are specific to a given aggregation level, which shows that management strategies should generally be tailored to each specific landscape. However, we also identified a strategy that is efficient for all levels of landscape aggregation. This one-size-fits-all strategy has important practical implications because of its simple applicability at a large scale.

Keywords: analytical and theoretical plant pathology, disease control and pest management, ecology and epidemiology, optimization, population biology, SEIR, sharka, spatiotemporal model, virology, virus
\end{abstract}

Understanding epidemiological processes is crucial to anticipate disease outbreaks, predict the spread of epidemics, and, thus, propose optimized management strategies that aim to reduce or eliminate a disease (Ferguson et al. 2001). However, epidemics are the result of complex interactions between biological processes, human interventions, and the spatial arrangement of patches in the landscape. Thus, understanding epidemics and assessing the effectiveness of disease management options is often a difficult task, especially because field trials are generally limited by regulatory, ethical, and logistical constraints (particularly for large-scale experimental studies). To overcome these limitations, epidemiological models are an interesting approach because of their ability to test several epidemic and management scenarios using the best available knowledge (Cunniffe et al. 2015a; Keeling and Rohani 2008; Keeling et al. 2003).

Spatially explicit models have been used to estimate epidemiological parameters such as dispersal functions (Parnell et al. 2011; Parry et al. 2014; Pleydell et al. 2018; Soubeyrand et al. 2008), infection rates (Cunniffe et al. 2014), and incubation durations (Cunniffe et al. 2014; Pleydell et al. 2018). This

${ }^{\dagger}$ Corresponding author: G. Thébaud; E-mail: gael.thebaud@inra.fr

Funding: This work was supported by a scholarship to C. Picard from Institut National de la Recherche Agronomique/Agence Nationale de Sécurité Sanitaire de l'Alimentation, de l'Environnement et du Travail, and by Institut National de la Recherche Agronomique (BEcOSMASH project, funded by the $\mathrm{SMaCH}$ Metaprogram and the SAE2 Department) and Agropolis Fondation (E-SPACE project).

*The $\boldsymbol{e}$-Xtra logo stands for "electronic extra" and indicates that six supplementary figures and two supplementary tables are published online.

The author(s) declare no conflict of interest.

(c) 2019 The American Phytopathological Society approach leads to disease-specific, data-calibrated models that can then be exploited to assess the efficacy of control measures; for example, sampling frequency and intensity (Parnell et al. 2012, 2014; Soubeyrand et al. 2018), plantation density (Chan and Jeger 1994; Cunniffe et al. 2014, 2015b; Jeger and Chan 1995), insecticide spraying frequency and location (Filipe et al. 2012), and zones and dates of removal (Cunniffe et al. 2014, 2015b; Filipe et al. 2012; Parnell et al. 2009, 2010; Sisterson and Stenger 2013).

However, these modeling studies mostly focused on only one or two management parameters, other parameters being set at their reference value. Rimbaud et al. (2019) did optimize several parameters simultaneously; however, like almost all previous studies, they performed simulations in a single landscape and did not consider landscape characteristics. Nevertheless, in order to study outbreaks and large-scale management strategies, considering landscape characteristics can be crucial. Indeed, they can influence epidemic dynamics, implying that the best management strategies may vary depending on the landscape (Papaix et al. 2014). A review by Ostfeld et al. (2005) analyzed the few studies that demonstrate how spatial locations of crop patches can influence disease risk, suggesting that a true integration of the landscape within epidemiological studies would be fruitful. As a consequence, promising approaches have been developed to integrate landscape characteristics into epidemiological models. For example, it was shown that, for the purpose of eradication, the optimum radius of orchard removals increases with the level of patch aggregation and the host density in the landscape, both factors increasing epidemic spread (Parnell et al. 2009, 2010). However, in these studies, patch layout is summarized by patch centroid coordinates although plot size and shape play an important role in disease dispersal (Mikaberidze et al. 2016; Pleydell et al. 2018) and, thus, on the impact of disease management. Indeed, such simplification can introduce a bias in connectivity estimates when patches have different shapes and sizes (e.g., the connectivity between the 
centroids of two patches would erroneously be the same whatever their area). Here, we try to understand how landscape structure influences disease spread and the impact of control options thanks to simulations of disease spread and management on various landscapes.

We apply this approach to sharka, one of the most damaging diseases of trees belonging to the Prunus genus (e.g., peach, apricot, and plum) (Cambra et al. 2006; Rimbaud et al. 2015). The causal agent of this disease, Plum pox virus (PPV, genus Potyvirus, family Potyviridae), is naturally transmitted by aphids in a nonpersistent manner. The presence of PPV symptoms such as fruit deformation (Németh 1986), apparition of light green rings, mosaic, mottling, and distortions on the leaves (Rimbaud et al. 2015) reduces potential sales, occasioning a significant economic impact (Cambra et al. 2006), with yield losses up to $100 \%$ for the most sensitive cultivars. Different alternatives for sharka management strategies exist in the world (eradication, suppression, containment, or resilience) depending on the epidemic context (Rimbaud et al. 2015). In France, sharka management aims to reduce the number of PPV-infected trees to mitigate its impact (suppression); it is compulsory and defined by a national decree specifying a complex procedure based on nursery protection, frequent visual inspections of orchards, and removal of symptomatic trees or, possibly, whole orchards, as well as plantation restrictions (JORF 2011) (Fig. 1). In a previous study, key parameters of a sharka epidemic were identified, and an improved management strategy was highlighted for a single landscape (Rimbaud et al. 2019). In the present article, we use the same model to analyze the influence of landscape characteristics on plant disease control. For that purpose, we first studied the influence of landscape structure on Prunus productivity under the French management strategy (JORF 2011). Next, we used sensitivity analyses to assess the relative influence of model parameters on crop productivity, depending on the level of patch aggregation in the landscape. Then, we exploited the results of these analyses to identify several efficient strategies and we studied how the landscape influences their impact. This last point allowed us to assess whether the management can be generic (i.e., if a unique management strategy is efficient for all landscapes) or should be specific to each landscape.

\section{MATERIALS AND METHODS}

Landscape generation. In this study, the landscape is considered as a set of cultivated patches (i.e., pieces of land) in a defined study area on which the pathogen may spread when trees are planted. Patches of different sizes and aggregation levels were simulated (i) by duplicating real patches and (ii) using a Ttessellation algorithm.

Duplication of real patches. In previous studies, Pleydell et al. (2018) and Rimbaud et al. (2018) developed a model allowing them to simulate virus dispersal on a real landscape comprising 553 patches ( 524 ha of patches in a study area of 2,730 ha). This landscape was generated from a database collected in a peachproducing area in southeastern France. Here, artificial landscapes were constructed by duplicating this real landscape three times to obtain a total of 1,659 patches and seven times to obtain 3,871 patches (with the size of the study area increasing accordingly). In addition, to obtain landscapes with lower levels of patch aggregation through a reduction in patch density, some of the patches were selected (subsampled) from the two duplicated landscapes. For the landscape duplicated three times, 60 and $30 \%$ of the patches were selected randomly from each of the original landscapes (with 553 patches). For the landscape duplicated seven times, 60 and $20 \%$ of the patches were selected randomly from each of the original landscapes. Three independent landscapes were generated for each subsampled landscape (Supplementary Fig. S1). In this way, we obtained three sizes of study area, with one aggregation level for the smaller one (corresponding to the real landscape), and three different aggregation levels for each of the larger study areas (and three different landscapes for each subsampled landscape).

Landscape simulations with T-tessellations. To avoid being dependent on a single real landscape, we also simulated new agricultural landscapes with various levels of patch aggregation and a realistic outlook (Fig. 2). Three landscapes comprising $n=400$ patches were simulated, hereafter called $\mathrm{H}, \mathrm{M}$, and L based on the value of the aggregation parameter: $\mathrm{d}=1$ (H: high aggregation), $\mathrm{d}=$ 200 (M: medium aggregation), and $d=400$ (L: low aggregation), and $d=400$ (L= low aggregation) (Fig. 3; Supplementary Fig. S2). These values were chosen to represent diverse patch aggregation levels: with $\mathrm{d}=400$, patches were scattered throughout the study window and with $d=1$, we obtained only neighboring patches in each cluster. The value of parameter $\mathrm{p}$ was chosen to ensure the simulation of, on average, 15 clusters: $\mathrm{p}=1-15 / n=0.96$. This parameter accounts for landscape irregularities due to the presence of features such as soil, topology, lakes, rivers, roads, or towns, which require that patches are generally grouped. We simulated 30 landscapes with these parameter values for each aggregation level.

Epidemiological model. To simulate disease spread and management in landscapes, we used an existing stochastic, spatially explicit, susceptible-exposed-infectious-removed (SEIR) model (Pleydell et al. 2018; Rimbaud et al. 2018, 2019). The model is patch-based, with a discrete time step of 1 week. At the beginning of the simulation, the trees in the patches are not infected: they are in the "susceptible" (i.e., healthy) state. The virus is introduced at the beginning of the first year of the simulation in one of the patches (defined by its connectivity quantile) and then spreads through orchards, causing changes in tree status: from "susceptible", they become "exposed" just after virus infection, "infectious hidden" (and symptomatic) after the end of the latent period, "infectious detected" after detection of the infected tree during surveys, and "removed" when the tree is removed from the patch. In addition, during the entire simulation new introductions can also occur (with a specified probability) at each patch plantation. Epidemic spread is governed by six epidemiological parameters (Table 1) that were estimated previously (Pleydell et al. 2018). Furthermore, a management strategy based on the French management of sharka in Prunus orchards is implemented as previously described (Rimbaud et al. 2019). Briefly, a disease management strategy defined by 23 parameters (Fig. 1; Supplementary Table S1) is applied after 5 years of epidemic to allow the spread of the virus. The model output is an economic criterion: the net present value (NPV), which corresponds to the sum of the gross margin (GM) calculated each year and updated by a discount rate (Rimbaud et al. 2019). The GM represents the difference between the benefits generated by the cultivation of productive hosts and the costs induced by production and management actions (including surveillance, removal, and replantation). To compare the different landscapes, GM (and, hence, NPV) were always divided by the total orchard area in the landscape.

Epidemic simulations and sensitivity analyses. Simulations with the French management. To study the influence of the landscape on productivity under the French management strategy, we performed simulations for all of the landscapes described above. A realistic turnover of peach orchards was simulated on patches using a mean cultivation duration of 15 years (Rimbaud et al. 2019). Simulations were run for 35 years (5 years without management and 30 years with management), which is a reasonable duration to assess the long-term impact of an epidemic in cultivated perennial plants. For each simulation, the six epidemiological parameters were drawn from uniform distributions using the bounds corresponding to sharka pathosystem (Rimbaud et al. 2019) (Table 1) and management parameters representing the French management strategy (Fig. 1) (JORF 2011). On the duplicated real landscapes, 10,000 simulations were carried out on the three landscapes without subsampled patches, and 3,334 simulations 
were performed on each of the three replicates of the subsampled landscapes (to obtain a total of 10,000 simulations for each aggregation level). Likewise, on landscapes simulated by the Ttessellation algorithm, 334 simulations were performed for each of the 30 replicates.

Sensitivity analyses. The relative influence of epidemic and management parameters on disease impact was assessed for simulated sharka epidemics. For this purpose, Sobol's method for sensitivity analysis was used, which consists of (i) the definition of target parameters and of their respective variation ranges; (ii) the generation of a numerical experimental design to explore parameter space; (iii) simulation; and (iv) the computation of Sobol's sensitivity indices, which quantify the influence of each target parameter on the output variable (Faivre et al. 2013; Saltelli et al. 2008; Sobol 1993). The first-order sensitivity index of a parameter $\left(\mathrm{SI}_{1}\right)$ measures the main effect of this parameter whereas the total sensitivity index $\left(\mathrm{SI}_{\text {tot }}\right)$ also accounts for its interactions with other parameters. These indices are bounded by 0 and 1; a total index

\section{Surveillance}

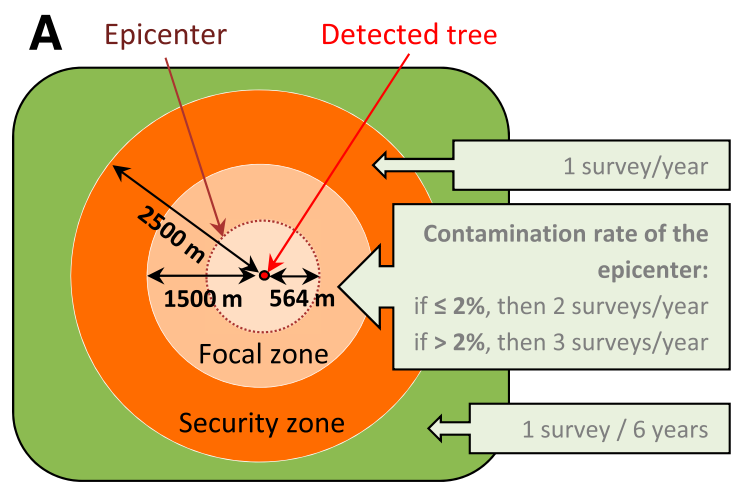

Surveillance zones established for 3 years

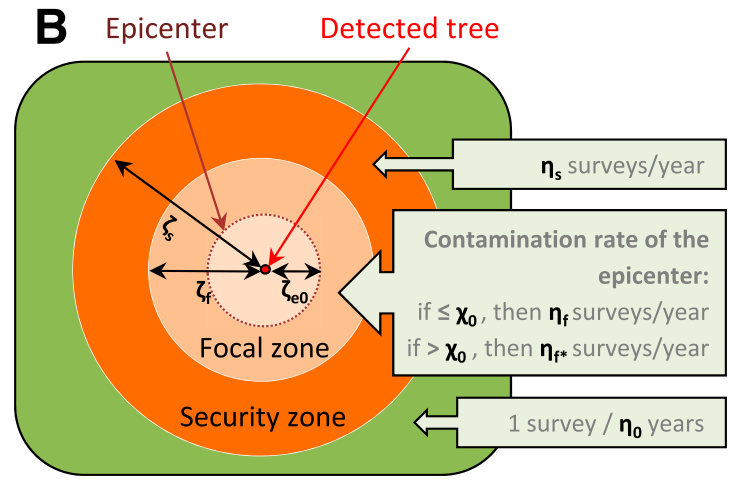

Surveillance zones established for $\gamma_{0}$ years

\section{Plantation / Young orchards}

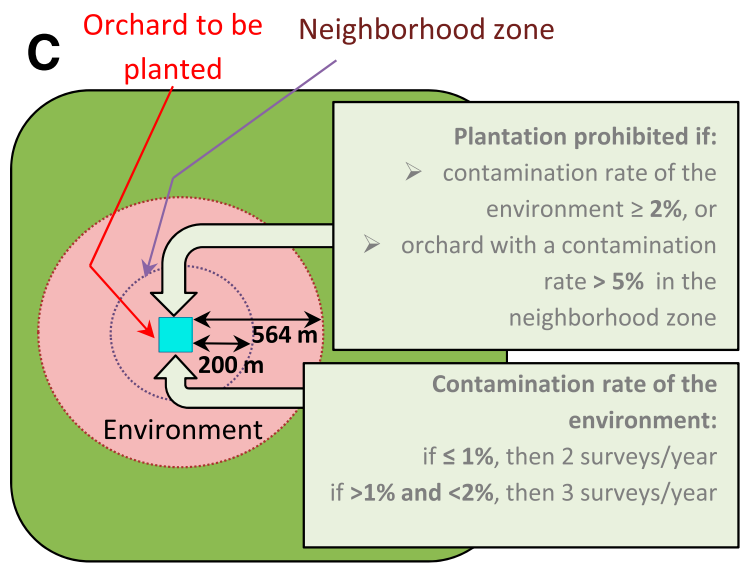

Surveillance zones established for 3 years

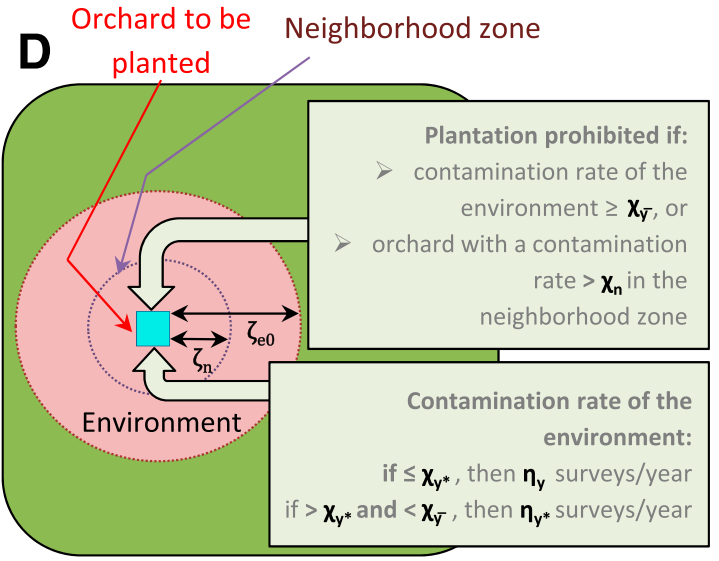

Surveillance zones established for $v_{y}$ years

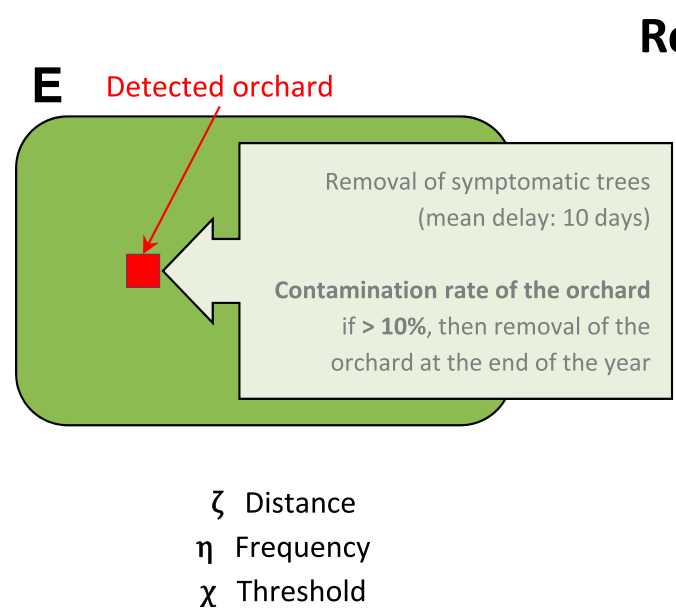

Removals

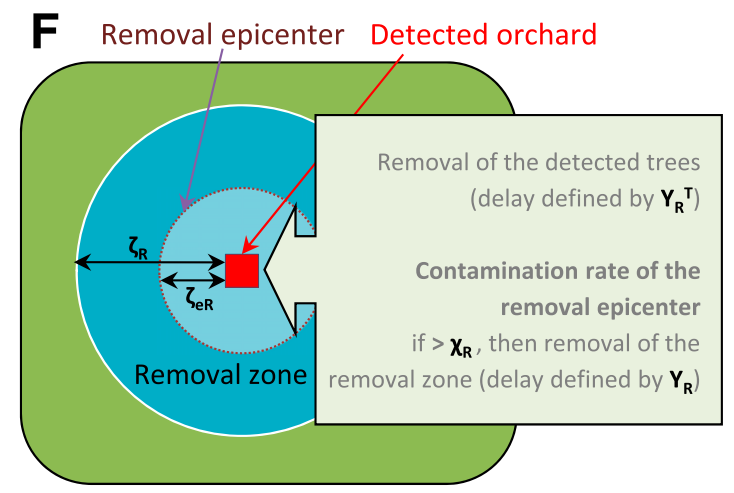

Delay before replantation of a removed orchard: $\gamma_{s}$ years

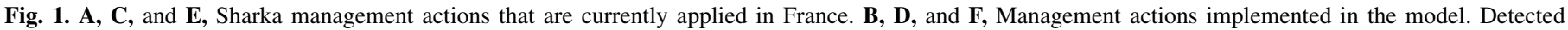
orchards include at least one observed infected tree. 
close to 0 indicates that the parameter has a negligible effect on the output variable.

Here, to get results specific to each level of patch aggregation, three sensitivity analyses were performed independently for the three simulated landscape types. We targeted 23 control parameters defining the implemented management strategy and 6 epidemiological parameters. Variation ranges were defined as their respective definition domain, possibly restricted using an expert's opinion when this domain was infinite (Table 1) (Rimbaud et al. 2019). For each of the 30 landscapes (for each aggregation level), simulations were performed with 310,155 parameter combinations generated with Sobol sequences (Sobol 1967, 1976). Then, Sobol's indices were calculated on the mean of the 30 replicates. First-order indices were estimated with the Sobol-Saltelli method (Saltelli et al. 2010; Sobol et al. 2007) whereas total indices were estimated with the Sobol-Jansen method (Jansen 1999; Saltelli et al. 2010).

Simulation of improved strategies. Using outputs of the sensitivity analyses, we identified an improved strategy for each aggregation level. This improved strategy corresponds to the parameter combination leading to the highest NPV among the 310,155 combinations. We call these strategies "best point $H$ ", "best point M", and "best point L" for each aggregation level (high, medium, and low, respectively). Then, 10,000 simulations were performed with these three management strategies for the three aggregation levels as described above.

We compared the mean NPV ( $\overline{\mathrm{NPV}}$ ) and the lowest decile of the NPV (i.e., $10 \%$ of the NPV values are below the lowest decile $\left.\left[\mathrm{NPV}_{10 \%}\right]\right)$. This last criterion was chosen considering that farmers do not accept a management strategy which can too often lead to a low NPV. The purpose of this initial step was to assess whether a strategy that is efficient in a particular landscape remains efficient on landscapes with different characteristics. This provided an overview of the influence of landscapes features on management strategies.

Improvement of the landscape-specific management strategies. The sensitivity analyses were carried out with 310,155 combinations of both epidemiological and management parameters. Thus, the three strategies "best point H", "best point M", and "best point L" were selected because they were effective for one epidemic (characterized by the six epidemiological parameters). However, other combinations of management parameters could have led to higher NPV with other epidemic parameters. Thus, to better assess the most promising disease management strategies, we used a stratified sampling procedure to
$\mathrm{H}$

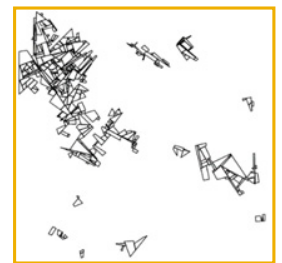

M

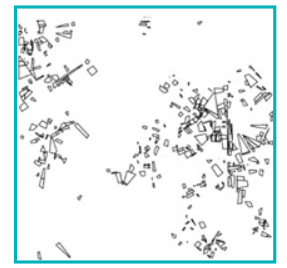

L

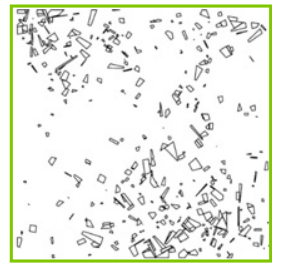

Fig. 3. Examples of landscapes simulated with a specified aggregation level for patches of susceptible hosts with parameters $n=400$ and $\mathrm{p}=15$. Three values of the aggregation parameter are used: $\mathrm{H}, \mathrm{d}=1$ (high aggregation); $\mathrm{M}$, $\mathrm{d}=200$ (medium aggregation); and $\mathrm{L}, \mathrm{d}=400$ (low aggregation).
Simulation of a complete tessellation with a T-tessellation algorithm

( $6.8 \times 6.8 \mathrm{~km}, 4936$ patches)

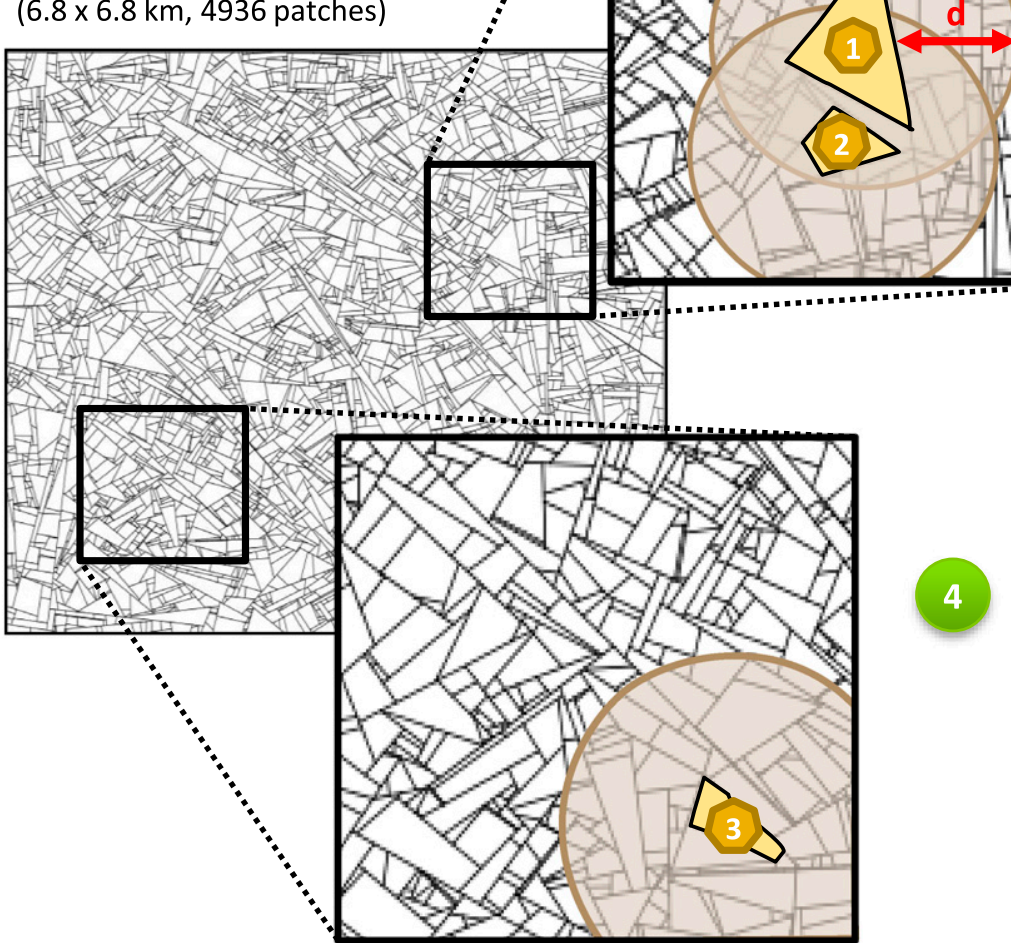

\section{2}

Random selection of a patch among the polygons (e.g., 1)

Definition of the
aggregation zone
(based on a
distance d)

Random selection of the next patch with probability :

- $\quad p$ in the aggregation zone (e.g., 2)

- 1-p: outside the aggregation zone (e.g., (3)

Fig. 2. Algorithm for the simulation of landscapes with a specified aggregation level for patches of susceptible hosts. The aggregation level is defined by three parameters: $n, p$, and d. Parameter d determines the size of an "aggregation zone", including all polygons located within d meters of a previously selected patch. In steps 2 and 4, patches are selected randomly and uniformly. The mean area of the simulated patches is equal to the mean area of the peach orchards composing a real landscape (0.95 ha). 
find improved combinations of management parameters for various "epidemic cases". Each epidemic case corresponds to a set of different value ranges of the epidemiological parameters (example of one epidemic case: $\mathrm{q}_{\kappa} \in[0,0.25], \beta \in[1.25,1.29], \phi \epsilon$ $[0.0046,0.0077], \mathrm{p}_{\mathrm{MI}} \in[0,0.05], \mathrm{W}_{\exp } \in[0.469,0.4865], \theta_{\exp } \epsilon$ $[1.71,1.925])$. The level of subdivision of the value ranges was based on the results of the sensitivity analyses, with more subdivisions for more influential epidemiological parameters. Then, each of the 310,155 parameter combinations was allocated to the corresponding epidemic case. Finally, for each epidemic case and each aggregation level, we identified the combination of management parameters leading to the highest NPV, and we performed 10,000 simulations with these strategies on the corresponding landscape, while varying the epidemiological parameters within their respective variation ranges.

To finish, we selected the 10 parameter combinations corresponding to the highest $\mathrm{NPV}_{10 \%}$ for each aggregation level and we performed 10,000 simulations of these strategies on the other landscapes. The strategies leading to the best $\mathrm{NPV}_{10 \%}$ for each aggregation level were called "improved strategy H", "improved strategy M", and "improved strategy L".

\section{RESULTS}

Landscape organization influences the impact of management strategies. Landscape influence on productivity with

TABLE 1. Epidemiological parameters implemented in the model and their variation ranges in simulations

\begin{tabular}{|c|c|c|c|}
\hline Parameter & Description & Minimum & Maximum \\
\hline $\mathrm{q}_{\kappa}$ & $\begin{array}{l}\text { Quantile of the connectivity of the } \\
\text { patch of first introduction }\end{array}$ & 0 & 1 \\
\hline$\phi$ & Probability of introduction at plantation & 0.0046 & 0.0107 \\
\hline $\mathrm{p}_{\mathrm{MI}}$ & $\begin{array}{l}\text { Relative probability of massive } \\
\text { introduction }\end{array}$ & 0 & 0.1 \\
\hline $\mathrm{W}_{\exp }$ & $\begin{array}{l}\text { Expected value of the dispersal } \\
\text { weighting variable }\end{array}$ & 0.469 & 0.504 \\
\hline$\beta$ & Transmission coefficient & 1.25 & 1.39 \\
\hline$\theta_{\text {exp }}$ & $\begin{array}{l}\text { Expected duration of the latent period } \\
\text { (years) }\end{array}$ & 1.71 & 2.14 \\
\hline
\end{tabular}

A

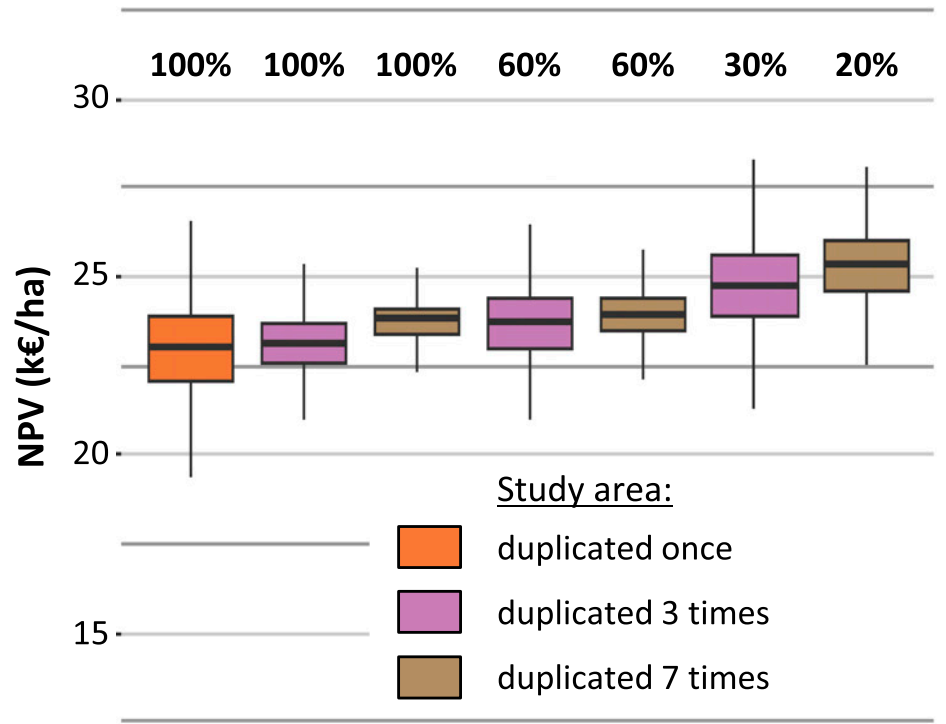

\section{Relative patch density}

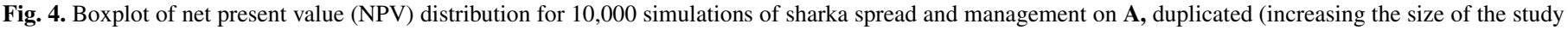
area) and subsampled (reducing patch density and aggregation level) real landscapes and $\mathbf{B}$, simulated landscapes with decreasing patch aggregation level. the French management strategy. We performed simulations of epidemic spread on duplicated and simulated landscapes under the French management strategy (JORF 2011). In both cases, the NPV increased for landscapes with decreasing patch aggregation (Fig. 4).

In order to understand why the NPV is affected in landscapes with higher patch aggregation, we observed disease prevalence through time and studied the impact of patch aggregation on components of the NPV for simulated epidemics (Supplementary Fig. S3). During the early years of the epidemic, the virus spread faster in landscape $\mathrm{H}$ than in landscapes $\mathrm{M}$ and $\mathrm{L}$; prevalence and incidence, therefore, were slightly higher. Thus, surveillance was strengthened and increased costs (inducing a lower GM). In addition, the increased number of removals led to a decrease in the number of productive trees (the average number of productive trees per hectare per year was 553,557 , and 559 for $\mathrm{H}, \mathrm{M}$, and L landscapes, respectively, over the 30 years of the epidemic), which entails yield losses.

Landscape influence on sensitivity to model parameters. One sensitivity analysis was performed for each of the three levels of patch aggregation on 23 management parameters and 6 epidemiological parameters in order to identify the most influential input parameters on the NPV (Fig. 5). We showed that two parameters related to plantation (contamination threshold for an orchard in the neighborhood, above which the plantation of orchards is forbidden $\left[\chi_{n}\right]$ ) and removals (contamination threshold in the removal epicenter, above which orchards inside the removal zone are removed $\left[\chi_{R}\right]$ ) have a strong influence on the NPV and this result does not depend on patch aggregation. The high impact of these parameters is likely due to a loss of productivity when the contamination threshold for the plantation bans and removal is too low.

However, although the two most influential parameters were the same for the three landscapes, their relative influence depends on landscape aggregation. For landscape $\mathrm{H}$, the most influential contributors to the NPV were first the plantation ban threshold $\left(\chi_{\mathrm{n}}\right.$; $\left.\mathrm{SI}_{\text {tot }}=0.62\right)$ and then the removal threshold $\left(\chi_{\mathrm{R}} ; \mathrm{SI}_{\text {tot }}=0.29\right)$. Conversely, for landscape $\mathrm{L}$, the most influential contributors to the NPV were first the removal threshold $\left(\chi_{R} ; \mathrm{SI}_{\text {tot }}=0.45\right)$ and then the plantation ban threshold $\left(\chi_{\mathrm{n}} ; \mathrm{SI}_{\text {tot }}=0.42\right)$. Overall, when the landscape is highly aggregated, much of the variance is explained by a few parameters; conversely, when the landscape is less

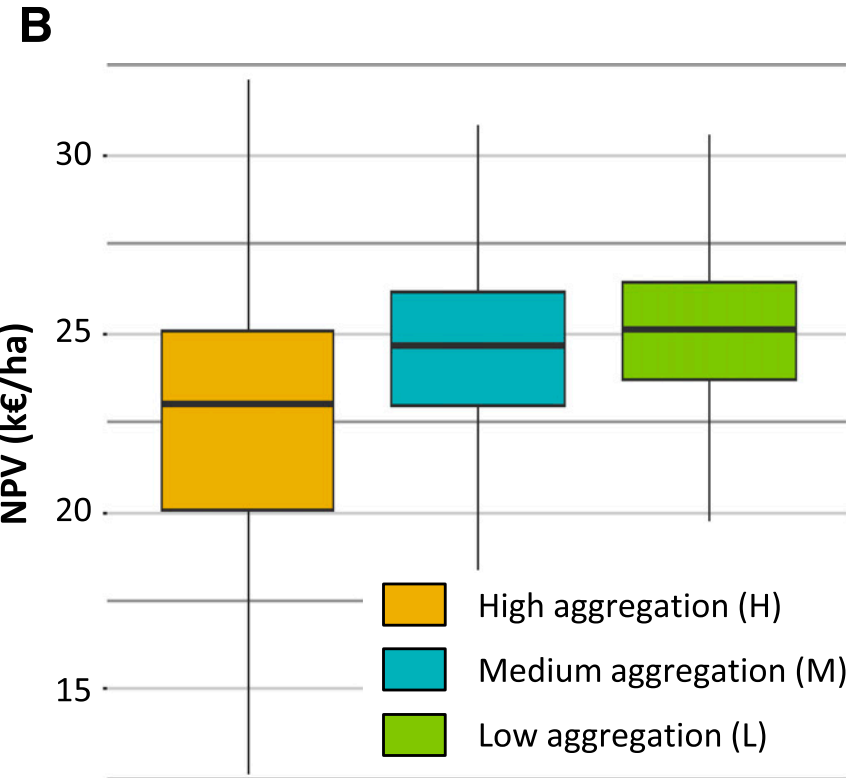


aggregated, a larger number of parameters explain the variance observed in the simulations (Fig. 5).

To summarize, management parameters do not have the same influence on the economic criterion depending on the landscape. Therefore, optimal management parameters can depend on landscape features.

Landscape influence on productivity for improved strategies. An improved strategy (i.e., the parameter combination resulting in the best NPV among the 310,155 tested combinations) was identified for each level of landscape aggregation (best point $\mathrm{H}$, best point $\mathrm{M}$, and best point $\mathrm{L}$ ). For the three aggregation levels, these management strategies only very rarely involved orchard plantation bans, and only symptomatic trees were removed (not entire orchards). In addition, surveillance zones (focal and security zones) were much smaller with these strategies than with the French management strategy (Supplementary Table S2), which reduces surveillance costs.

Then, simulations were carried out with these three strategies by varying epidemiological parameters on all of the simulated landscapes (Fig. 6). Simulations performed on a landscape with the parameter combination identified for the same landscape led to better $\overline{N P V}$ and $\mathrm{NPV}_{10 \%}$ than with the modeled French management strategy. In addition, these analyses showed that a management strategy that is efficient in a given landscape is not necessarily efficient in another one, and can be less profitable than the French strategy. Indeed, in our simulations, the best point $\mathrm{H}$ strategy was more profitable than the French strategy when applied on landscapes $\mathrm{M}$ and $\mathrm{L}$; however, the best point $\mathrm{M}$ and best point $\mathrm{L}$ strategies were less profitable than the French management strategy for landscape H; $\overline{\mathrm{NPV}}$ and $\mathrm{NPV}_{10 \%}$ were largely lower (i.e., risktaking is higher) than with the French strategy.

Landscape influence on improved management strategies. The sensitivity analyses showed that two of the six epidemiological parameters had a high impact on the NPV (Fig. 5): the quantile of the connectivity of the patch of first introduction $\left(\mathrm{q}_{\kappa}\right)$ and the transmission coefficient $(\beta)$. To define the epidemic cases (i.e., subsets of parameter values corresponding to similar epidemics), we divided the value ranges of these two parameters into four equal parts, and the four other epidemiological parameters $\left(\Phi, \mathrm{p}_{\mathrm{MI}}, \mathrm{W}_{\mathrm{exp}}, \theta_{\mathrm{exp}}\right)$ were divided into two equal parts. We obtained $4 \times 4 \times 2 \times 2 \times 2 \times 2=256$ epidemic cases for each level of landscape aggregation and, for each case, we identified the combination of management parameters leading to the highest NPV. The majority of these strategies did not involve orchard plantation bans (in $85 \%$ of the cases for landscape $\mathrm{H}$ and $89 \%$ for landscapes $\mathrm{M}$ and $\mathrm{L}$ ) and did not impose removal of entire orchards (in $68 \%$ of the cases for landscape $\mathrm{H}$ and $74 \%$ for landscapes $\mathrm{M}$ and $\mathrm{L}$ ). In addition, surveillance zones were again much smaller for these strategies than for the French management.

For each aggregation level, (i) simulations were carried out with the corresponding 256 strategies and (ii) the 10 parameter combinations resulting in the best $\mathrm{NPV}_{10 \%}$ were retained. Simulations were then performed with these 30 combinations on all of the landscapes (Supplementary Fig. S4). We observed that the impact of these management strategies was more important for landscape $H$ than for landscapes $\mathrm{M}$ and $\mathrm{L}$. Indeed, with the 30 management strategies, the $\mathrm{NPV}_{10 \%}$ varied between 15,945 and $22,987 € /$ ha for landscape $\mathrm{H}$ between 23,110 and $24,202 € /$ ha for landscape $M$, and between 23,111 and $24,616 € /$ ha for landscape $\mathrm{L}$. The strategies leading to the best $\mathrm{NPV}_{10 \%}$ (improved strategy $\mathrm{H}$, improved strategy $\mathrm{M}$, and improved strategy L) are depicted in Supplementary Fig. S5. We note that the strategy leading to the best $\mathrm{NPV}_{10 \%}$ was also the strategy leading to the best $\overline{\mathrm{NPV}}$ (for landscape L) or leading to a very close value to the best $\overline{\mathrm{NPV}}$ (for landscapes $\mathrm{H}$ and $\mathrm{M}$ ).

Finally, we compared the NPV from simulations without management, with the French management strategy, with the three best point strategies, and with the three improved strategies leading to the best NPV $10 \%$ (Fig. 6). We could find substantially improved NPV for the three levels of patch aggregation. For instance, in landscape $\mathrm{H}$ the $\mathrm{NPV}_{10 \%}$ was $17,652 € /$ ha with the French management strategy, $20,474 € /$ ha with strategy best point $H$, and $22,987 € /$ ha with improved strategy $\mathrm{H}$. In addition, although each landscape had a specific improved strategy, our simulations suggested that the improved strategy $\mathrm{H}$ could be an acceptable

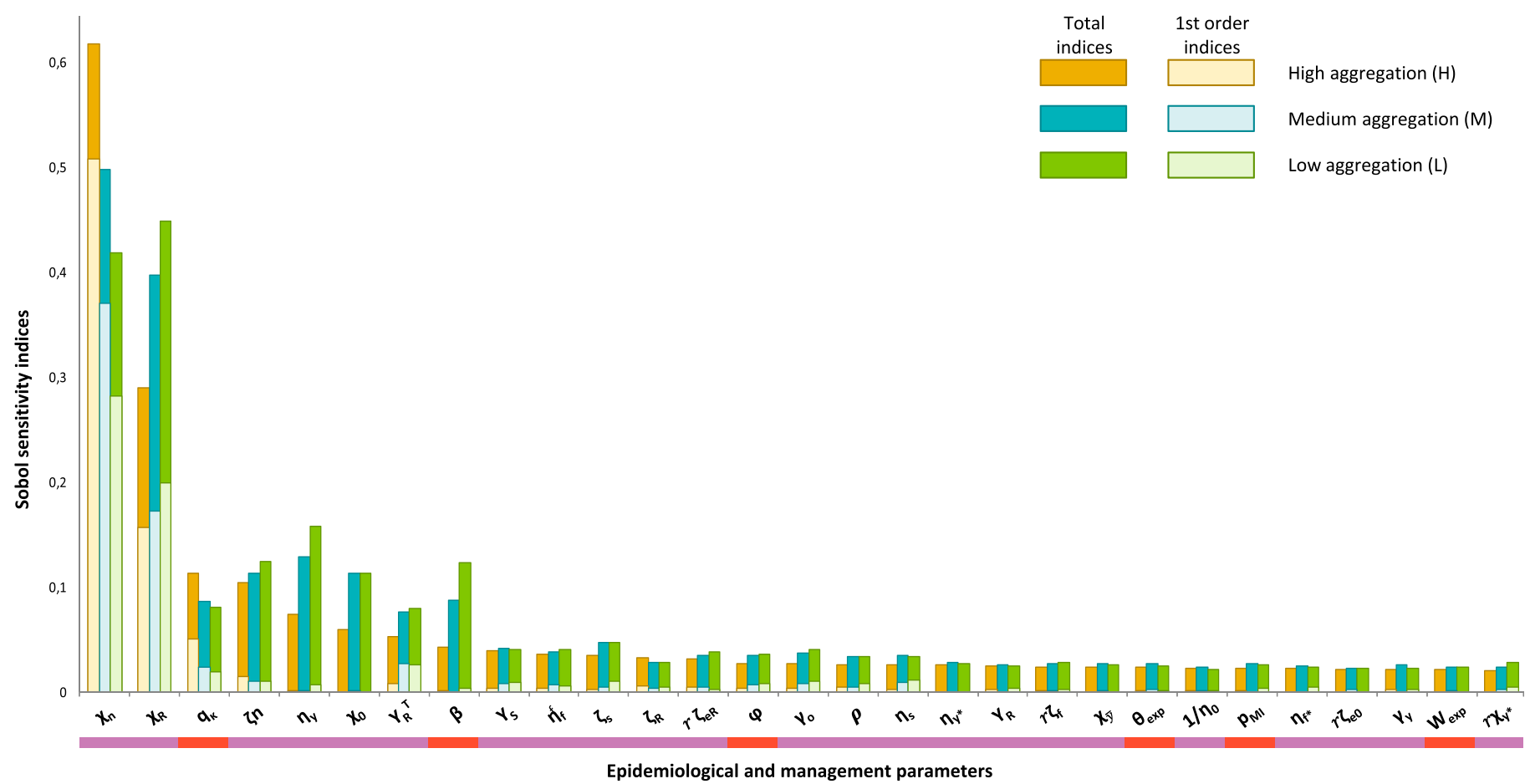

Fig. 5. Sobol sensitivity indices of 23 control parameters and 6 epidemiological parameters for the mean net present value ( $\overline{\mathrm{NPV}})$ assessed for sharka epidemics in landscapes with three different aggregation levels (high, medium, and low). The line under the figure indicates the epidemiological parameters (darker) and management parameters (lighter). 
compromise for all landscapes (Fig. 6). Indeed, application of the improved strategy $\mathrm{H}$ (Fig. 7) on landscapes $\mathrm{M}$ and $\mathrm{L}$ (instead of their respective improved strategies) led to a reduction of only 184 and $640 € /$ ha, respectively, in NPV $10 \%$ over 30 years.

\section{DISCUSSION}

This work aimed to understand how patch aggregation influences disease spread and the impact of control options. Simulations of disease spread and management within a sensitivity analysis framework showed that the landscape influences the profitability of different strategies for sharka control in peach orchards. In addition, the results of these sensitivity analyses were exploited to identify efficient strategies (more profitable, in the simulations, than the present French management of sharka). These strategies were efficient for a specific aggregation level but we also identified a generic strategy, namely the improved strategy $\mathrm{H}$, that was efficient for various levels of landscape aggregation.

Influence of landscape in modeling studies. Our study shows the importance of taking landscape characteristics into account in the design and optimization of disease management strategies. First, we showed that landscape aggregation influences sharka dispersal: in our simulations, profitability (NPV) increased with the distance between patches, both without sharka management or under the French management strategy (Fig. 6). In addition, we showed that landscape aggregation influences the impact of management strategies, both because the relative influence of the management parameters on the NPV depends on landscape aggregation (Fig. 5) and because a management strategy which is efficient for a landscape is not necessarily efficient for the other landscapes (Fig. 6). This demonstration that the efficiency of a disease management strategy depends on landscape aggregation has important consequences for the improvement of management strategies (or, maybe less realistically, for the optimization of the landscape itself).

This result also means that such studies must be based on either real or realistic landscapes. However, as pointed out above, generic realistic landscapes are rarely considered in epidemiological modeling studies. Because generic conclusions cannot be drawn on a single real landscape, it was important to simulate landscapes with a specified level of aggregation. Thus, we devised an algorithm based on T-tessellations to generate landscapes composed of various patches (with sufficiently realistic shapes and sizes) that were more or less aggregated. Disease dispersal and the impact of control options might also be influenced by other landscape structures such as mountains, lakes, rivers, forests, or roads (Brunker et al. 2018); or species composition or proportion of suitable habitat (Ostfeld et al. 2005), including the proportion of resistant versus susceptible hosts (Papaïx et al. 2014). Here, we chose to focus on patch aggregation but other landscape features might enter such models in the future if their epidemiological and economic impact is properly estimated.

In silico improvement of disease management. In the second part of the present study, for each level of landscape aggregation, we searched improved management strategies. This was challenging because we attempted to improve a complex strategy that included 23 management parameters (epidemiological modeling studies generally optimize 1 or 2 parameters at a time). To succeed, we used the results of sensitivity analyses for which numerous parameter combinations were tested. In addition, contrary to previous studies that pursued the same goal using an epidemiological criterion (Chan and Jeger 1994; Cunniffe et al. 2014, 2015b; Filipe et al. 2012; Jeger and Chan 1995; Parnell et al. 2009, 2010, 2012, 2014; Sisterson and Stenger 2013), here, we balanced all costs and benefits of disease management strategies within an economic criterion (Rimbaud et al. 2019), which is important when several parameters expressed in different units are cooptimized. Furthermore, modeling studies generally aim to improve the mean of the optimization criterion and do not take into account the level of risk aversion of decision-makers. However, as shown by Cunniffe et al. (2015b, 2016), the optimal strategy can depend on which percentile of a criterion is optimized. Because decision-makers generally tend to minimize the risk of devastating scenarios, here, we searched

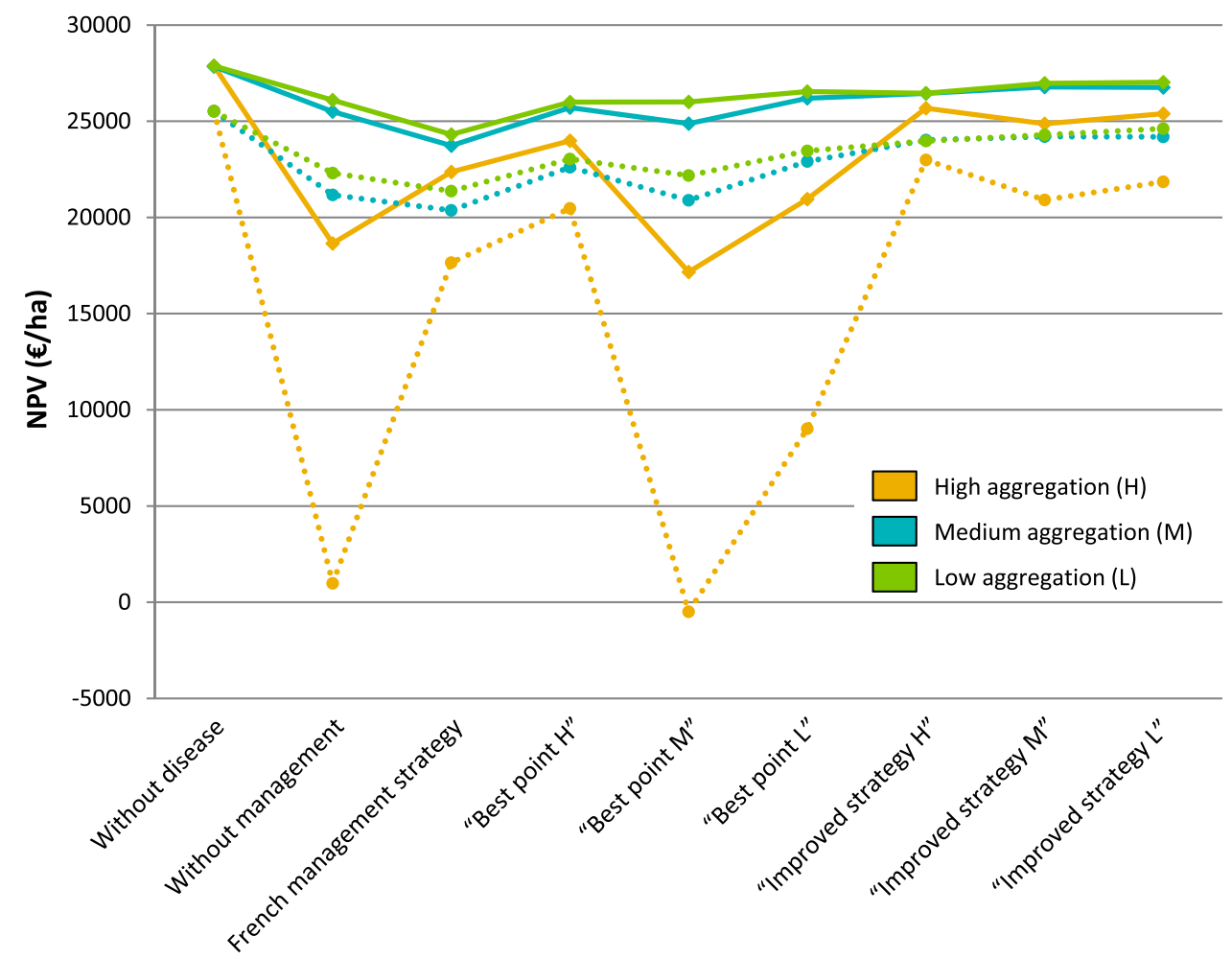

Fig. 6. Mean net present value (NPV) (solid lines) and NPV values below the lowest decile (dotted lines) obtained after simulations of Plum pox virus dispersal and management. 
efficient strategies on the basis of the lowest decile of the NPV (i.e., $\mathrm{NPV}_{10 \%}$ ). Improving this criterion allowed us to select management strategies that limit the proportion of epidemics causing substantial economic damage.

For different levels of patch aggregation, we identified different improved management strategies with our simulations (improved strategies $\mathrm{H}, \mathrm{M}$, and L). Applying these strategies on the corresponding landscapes, we obtained better $\overline{\mathrm{NPV}}$ (as well as $\mathrm{NPV}_{10 \%}$ ) than with previously improved strategies (Rimbaud et al. 2019) (Supplementary Fig. S6). This may be due to the fact that these previous strategies were improved for a unique landscape and lacked robustness to changes in landscape aggregation. In addition, the number of simulations performed for each strategy may influence the results. Here, we selected 256 candidate management strategies, for which we carried out 10,000 simulations where the epidemiological parameters varied, and we selected the strategy associated with an accurate estimate of the best $\mathrm{NPV}_{10 \%}$. In their study, Rimbaud et al. (2019) performed only 30 simulations for each of 310,155 random management strategies and (i) they isolated the parameter combination associated with the highest estimated NPV (best-value strategy) and (ii) they performed a marginal approximation of the optimal parameter set using the mode of the distribution of each parameter for the combinations associated with the $1 \%$ best NPVs (best-percent strategy). Our own attempt to perform such marginal approximation (not shown) failed to produce good NPV values, probably because the substantial interactions between management parameters (Fig. 5) are ignored by this approach.

\section{Surveillance}

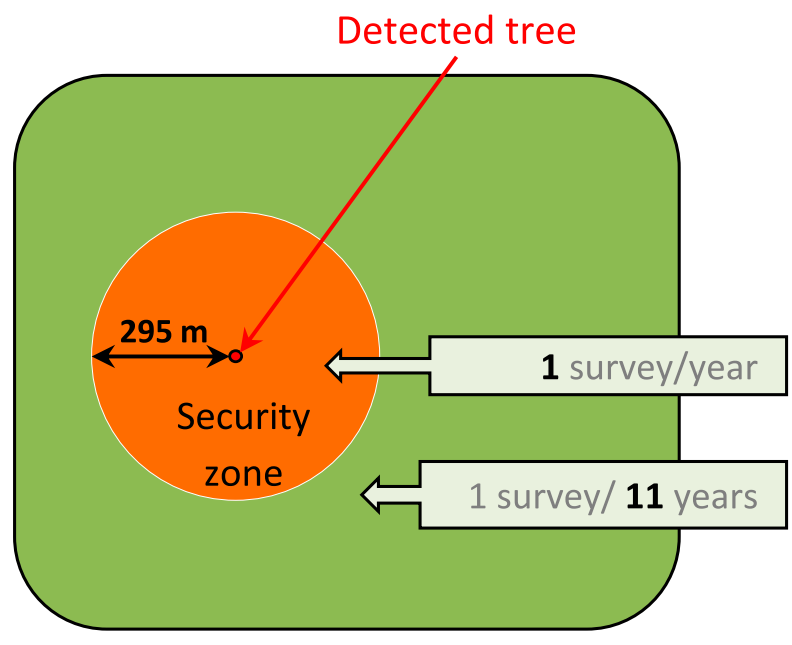

Surveillance zones established for $\mathbf{2}$ years

\section{Removals}

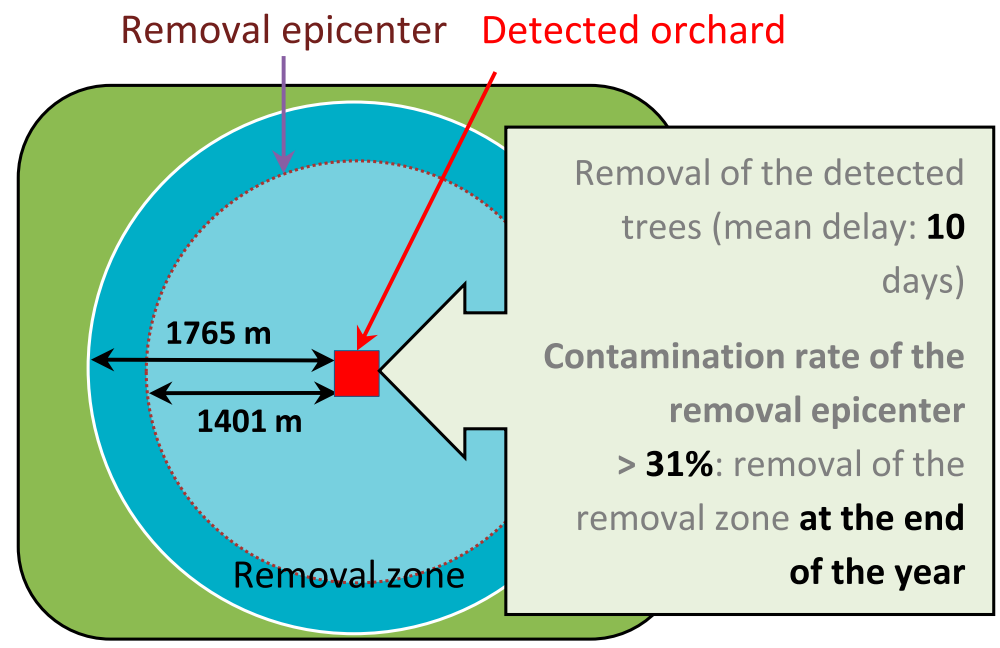

Delay before replantation of a removed orchard: 7 years

\section{Plantation / Young orchards}

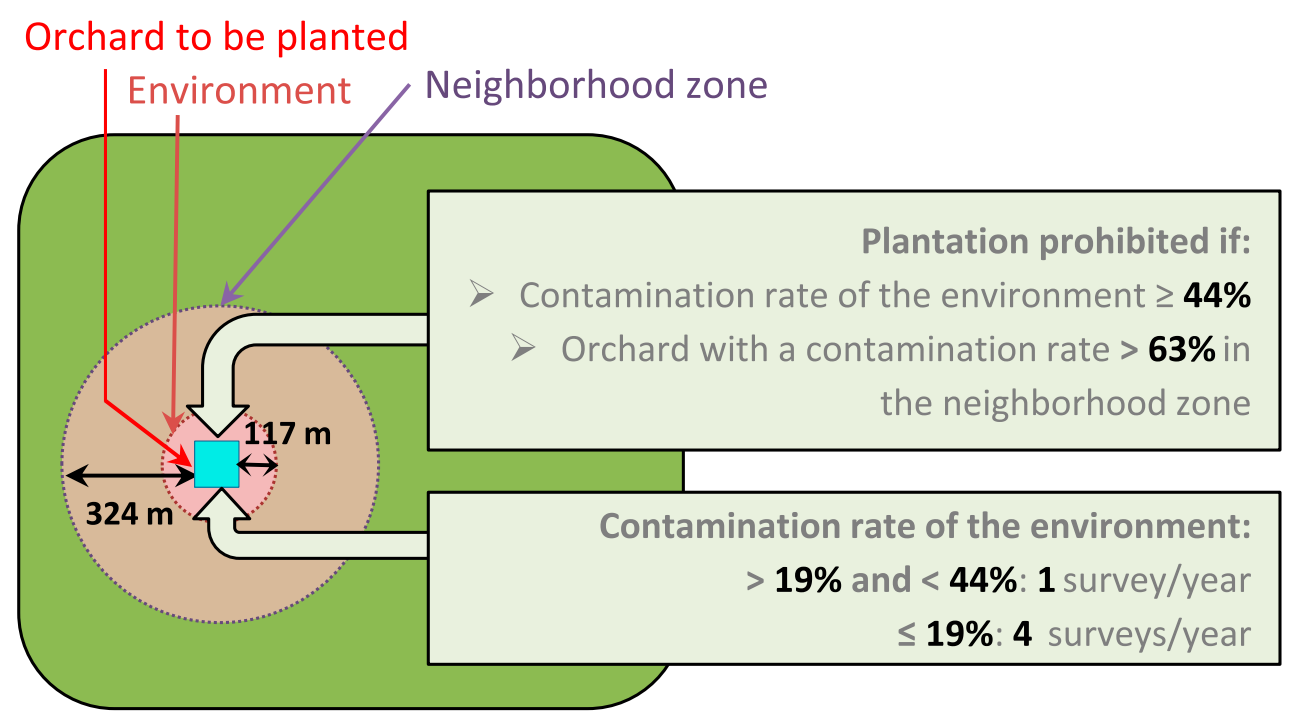

Surveillance zones established for $\mathbf{1}$ year

Fig. 7. Management actions for the improved strategy $\mathrm{H}$ (leading to the best lowest decile for the most aggregated landscape). 
Our results mean that, in theory, management could be tailored to each landscape. However, in practice, stakeholders may struggle to delineate zones that differ by their level of landscape aggregation, and to apply different strategies within the territory where they are involved. In addition, landscapes change through time, which means that strategies that are too specific to a given level of aggregation may become obsolete. Thus, such landscape-specific strategies may only be applicable when production areas with very different levels of landscape aggregation are distant enough. A practically useful alternative to such landscape-specific strategies is the identification of a robust, one-size-fits-all strategy which could be an efficient compromise for all of the landscapes. This is the case for the improved strategy $\mathrm{H}$ which, thus, may be applied at a wide scale. This strategy could be interesting for stakeholders because it is both more profitable and simpler to implement than the present French management strategy. Indeed, it requires surveillance of small areas around each detected tree, very rarely involves orchard plantation bans, and almost never imposes the removal of entire orchards (we note that the last two points correspond to the most influential parameters in the sensitivity analyses).

This work is relevant to stakeholders because it shows that both landscape-specific and landscape-generic disease management strategies can be identified and improved in silico. Indeed, the current strategy applied in France on 11,045 ha of peach orchard (Agreste 2013) reduces economic losses in case of severe sharka epidemics but, according to our simulations, on average, 36 million $€$ could be saved by using the improved strategy $\mathrm{H}$ over a period of 30 years for landscape $H$ ( 24 million $€$ for landscape $L)$ and 59 million $€$ for the lowest decile of the NPV (29 million $€$ for landscape L).

However, as previously mentioned (Rimbaud et al. 2019), our results can be affected by some model assumptions (for instance, the detection probability may be overestimated). In addition, we used a Sobol-type sensitivity analysis to improve management strategies. Although this analysis has good space-filling properties that enabled us to test a huge number of parameter combinations well spread throughout the parameter space (Sobol 1976), this one was so vast that better strategies can be found between the sampled points. The main goal of the present study was to explore the impact of landscape aggregation on improved disease management strategies but, if interest lies in approaching more closely the actual optimum, one option may be to explore the parameter space by iterative sensitivity analyses, as previously done (Rimbaud et al. 2019). However, this approach involves some arbitrary choices at each iteration and inefficiencies in the use of computing resources; thus, dedicated optimization algorithms may be more efficient for future work.

\section{ACKNOWLEDGMENTS}

We thank J. Papaï for advising on landscape simulations and S. Dallot for helpful feedback throughout the work. We acknowledge the CIRADUMR AGAP HPC Data Center of the South Green Bioinformatics platform (http://www.southgreen.fr), and we are grateful to Sébastien Ravel for the associated help.

\section{LITERATURE CITED}

Agreste. 2013. Inventaire des Vergers 2013. http://agreste.agriculture.gouv.fr/ enquetes/productions-vegetales-528/vergers-et-fruits/

Brunker, K., Lemey, P., Marston, D. A., Fooks, A. R., Lugelo, A., Ngeleja, C., Hampson, K., and Biek, R. 2018. Landscape attributes governing local transmission of an endemic zoonosis: Rabies virus in domestic dogs. Mol. Ecol. 27:773-788.

Cambra, M., Capote, N., Myrta, A., and Llácer, G. 2006. Plum pox virus and the estimated costs associated with sharka disease. EPPO Bull. 36: 202-204.

Chan, M. S., and Jeger, M. J. 1994. An analytical model of plant virus disease dynamics with roguing and replanting. J. Appl. Ecol. 31:413-427.

Cunniffe, N. J., Cobb, R. C., Meentemeyer, R. K., Rizzo, D. M., and Gilligan, C. A. 2016. Modeling when, where, and how to manage a forest epidemic, motivated by sudden oak death in California. Proc. Natl. Acad. Sci. U.S.A. 113:5640-5645.

Cunniffe, N. J., Koskella, B., Metcalf, C. J. E., Parnell, S., Gottwald, T. R., and Gilligan, C. A. 2015a. Thirteen challenges in modelling plant diseases. Epidemics 10:6-10.

Cunniffe, N. J., Laranjeira, F. F., Neri, F. M., DeSimone, R. E., and Gilligan, C. A. 2014. Cost-effective control of plant disease when epidemiological knowledge is incomplete: Modelling Bahia bark scaling of citrus. PLOS Comput. Biol. 10:e1003753.

Cunniffe, N. J., Stutt, R. O. J. H., DeSimone, R. E., Gottwald, T. R., and Gilligan, C. A. 2015b. Optimising and communicating options for the control of invasive plant disease when there is epidemiological uncertainty. PLOS Comput. Biol. 11:e1004211.

Faivre, R., Ioos, B., Mahévas, S., Makowski, D., and Monod, H. 2013. Analyse de Sensibilité et Exploration de Modèles. Editions Quae, Versailles, France.

Ferguson, N. M., Donnelly, C. A., and Anderson, R. M. 2001. Transmission intensity and impact of control policies on the foot and mouth epidemic in Great Britain. Nature 413:542-548.

Filipe, J. A., Cobb, R. C., Meentemeyer, R. K., Lee, C. A., Valachovic, Y. S., Cook, A. R., Rizzo, D. M., and Gilligan, C. A. 2012. Landscape epidemiology and control of pathogens with cryptic and long-distance dispersal: Sudden oak death in northern Californian forests. PLOS Comput. Biol. 8: e1002328.

Jansen, M. J. W. 1999. Analysis of variance designs for model output. Comput. Phys. Commun. 117:35-43.

Jeger, M. J., and Chan, M. S. 1995. Theoretical aspects of epidemics: Uses of analytical models to make strategic management decisions. Can. J. Plant Pathol. 17:109-114.

JORF. 2011. Arrêté du 17 mars 2011 relatif à la lutte contre le Plum Pox Virus, agent causal de la maladie de la Sharka, sur les végétaux sensibles du genre Prunus. République Française. JORF no. 0067 du 20 mars 2011. Paris, France. https://www.legifrance.gouv.fr/affichTexte.do?cidTexte= JORFTEXT000023728913\&dateTexte $=\&$ categorieLien $=$ id

Keeling, M. J., and Rohani, P. 2008. Modeling Infectious Diseases in Humans and Animals. Princeton University Press, Princeton, NJ, U.S.A.

Keeling, M. J., Woolhouse, M. E. J., May, R. M., Davies, G., and Grenfell, B. T. 2003. Modelling vaccination strategies against foot-and-mouth disease. Nature 421:136-142.

Mikaberidze, A., Mundt, C. C., and Bonhoeffer, S. 2016. Invasiveness of plant pathogens depends on the spatial scale of host distribution. Ecol. Appl. 26: 1238-1248.

Németh, M. V. 1986. Plum pox (Sharka). Pages 463-479 in: Virus, Mycoplasma and Rickettsia Diseases of Fruit Trees. Akadémiai Kaidó, Budapest, Hungary.

Ostfeld, R. S., Glass, G. E., and Keesing, F. 2005. Spatial epidemiology: An emerging (or re-emerging) discipline. Trends Ecol. Evol. 20:328-336.

Papaix, J., Adamczyk-Chauvat, K., Bouvier, A., Kiêu, K., Touzeau, S., Lannou, C., and Monod, H. 2014. Pathogen population dynamics in agricultural landscapes: The Ddal modelling framework. Infect. Genet. Evol. 27:509-520.

Parnell, S., Gottwald, T. R., Gilks, W. R., and van den Bosch, F. 2012. Estimating the incidence of an epidemic when it is first discovered and the design of early detection monitoring. J. Theor. Biol. 305:30-36.

Parnell, S., Gottwald, T. R., Gilligan, C. A., Cunniffe, N. J., and van den Bosch, F. 2010. The effect of landscape pattern on the optimal eradication zone of an invading epidemic. Phytopathology 100:638-644.

Parnell, S., Gottwald, T. R., Irey, M. S., Luo, W., and van den Bosch, F. 2011. A stochastic optimization method to estimate the spatial distribution of a pathogen from a sample. Phytopathology 101:1184-1190.

Parnell, S., Gottwald, T. R., Riley, T., and van den Bosch, F. 2014. A generic risk-based surveying method for invading plant pathogens. Ecol. Appl. 24: 779-790.

Parnell, S., Gottwald, T. R., van den Bosch, F., and Gilligan, C. A. 2009. Optimal strategies for the eradication of Asiatic citrus canker in heterogeneous host landscapes. Phytopathology 99:1370-1376.

Parry, M., Gibson, G. J., Parnell, S., Gottwald, T. R., Irey, M. S., Gast, T. C., and Gilligan, C. A. 2014. Bayesian inference for an emerging arboreal epidemic in the presence of control. Proc. Natl. Acad. Sci. U.S.A. 111: 6258-6262.

Pleydell, D. R. J., Soubeyrand, S., Dallot, S., Labonne, G., Chadœuf, J., Jacquot, E., and Thébaud, G. 2018. Estimation of the dispersal distances of an aphid-borne virus in a patchy landscape. PLOS Comput. Biol. 14: e1006085.

Rimbaud, L., Bruchou, C., Dallot, S., Pleydell, D. R. J., Jacquot, E., Soubeyrand, S., and Thébaud, G. 2018. Using sensitivity analysis to identify key factors for the propagation of a plant epidemic. R. Soc. Open Sci. 5:171435.

Rimbaud, L., Dallot, S., Bruchou, C., Thoyer, S., Jacquot, E., Soubeyrand, S., and Thébaud, G. 2019. Improving management strategies of plant diseases using sequential sensitivity analyses. Phytopathology 109:1184-1197. 
Rimbaud, L., Dallot, S., Gottwald, T., Decroocq, V., Jacquot, E., Soubeyrand, S., and Thébaud, G. 2015. Sharka epidemiology and worldwide management strategies: Learning lessons to optimize disease control in perennial plants. Annu. Rev. Phytopathol. 53:357-378.

Saltelli, A., Annoni, P., Azzini, I., Campolongo, F., Ratto, M., and Tarantola, S. 2010. Variance based sensitivity analysis of model output. Design and estimator for the total sensitivity index. Comput. Phys. Commun. 181: 259-270.

Saltelli, A., Ratto, M., Andres, T., Campolongo, F., Cariboni, J., Gatelli, D., Saisana, M., and Tarantola, S. 2008. Global Sensitivity Analysis: The Primer. Wiley, Chichester, England.

Sisterson, M. S., and Stenger, D. C. 2013. Roguing with replacement in perennial crops: Conditions for successful disease management. Phytopathology 103:117-128.

Sobol, I. M. 1967. On the distribution of points in a cube and the approximate evaluation of integrals. USSR Comput. Math. Math. Phys. 7:86-112.
Sobol, I. M. 1976. Uniformly distributed sequences with an additional uniform property. USSR Comput. Math. Math. Phys. 16:236-242.

Sobol, I. M. 1993. Sensitivity estimates for nonlinear mathematical models. Math. Modell. Comput. Exp. 1:407-414.

Sobol, I. M., Tarantola, S., Gatelli, D., Kucherenko, S. S., and Mauntz, W. 2007. Estimating the approximation error when fixing unessential factors in global sensitivity analysis. Reliab. Eng. Syst. Saf. 92: 957-960.

Soubeyrand, S., de Jerphanion, P., Martin, O., Saussac, M., Manceau, C., Hendrikx, P., and Lannou, C. 2018. Inferring pathogen dynamics from temporal count data: The emergence of Xylella fastidiosa in France is probably not recent. New Phytol. 219:824-836.

Soubeyrand, S., Held, L., Höhle, M., and Sache, I. 2008. Modelling the spread in space and time of an airborne plant disease. J. R. Stat. Soc. C $57: 253-272$ 